\section{OBSERVATIONS ON THE NATURE OF CANCEROUS GROWTHS.}

$\mathrm{M} \mathrm{UCH}$ of the advance made within recent years in our knowledge of the nature of disease can fairly be traced to the general recognition of the principle that pathological problems can be resolved in the first instance into cell problems. Not only is the modern practice of aseptic surgery founded on this principle, but it also forms the basis of rational as opposed to merely empirical therapeutics.

It is not, however, always easy to interpret the evidence drawn from a study of the cells, for though they may be described as the units of bodily organisation they are themselves extremely complex. Thus it happens that the explanation of this or that series of phenomena is often reached by a roundabout route.

Amongst the diseases in which the cellular aspects of the case thrust themselves prominently into the foreground, few are perhaps more remarkable than those malignant tumours popularly grouped under the general term of cancer. These growths are very numerous in character, they appear in widely different regions of the body, and they produce more or less profound disturbances in the organism in which they occur. They are not restricted to mankind, but, as means of investigation are improved, they are shown to afflict members of very different groups of animals included in the Vertebrata, and it may be expected that they will probably be identified in invertebrate animals also when a systematic search is made for them.

But however diverse these growths may be, both as regards the animals in which they occur and in the gross structure which they exhibit, they nevertheless present one important feature in common. They all essentially consist of cells that are multiplying in a manner uncoordinated with the requirements or advantages of the remaining cells and tissues of the animal affected. The growth as a whole behaves as a parasite-a foreign organism which lives at the expense of, and exercises a destructive influence on, the cells of the normal tissues it invades. The growth itself also betrays, in a greater or less degree, an organisation of its own, and its cells can commonly be readily distinguished from those of the host on which it preys. This independence on the part of the malignant growth has long been recognised, and it is often coupled with a power of dispersal in the body that produces new centres of infection and consequent spread of the disease. This is especially clearly shown in the case of a carcinomatous growth in mice, which can, as Jensen has shown, be transferred to the bodies of other mice by inoculation, and his results have been abundantly confirmed by Bashford and Murray in this country.

Although the cellular symptoms are for the most part not difficult to recognise, the causes that determine the origin of the neoplasm are still to seek. It is clear, however, that they operate in producing some change in cells that previously were not distinguished from those other units of which the body is composed.

Numerous theories and hypotheses have, from time to time, been advanced to account for the phenomena, but it is only when the nature of the altered structure itself is understood that we can expect to be in a position to investigate seriously the nature of the causes that produce it, and thence to bring the latter under control.

It has been suggested that a micro-organism is concerned in the production of some toxin that is more directly responsible for the mischief. Sporozoa, yeasts, psorosperms, and bacteria, have at different times been identified as the exciting causes, but careful examinNO. 1788 , vOL. 69 ] ation has failed to confirm these statements; whilst in a number of cases it is certain that normal cell constituents themselves have been mistaken for the supposed parasites. A modification of the parasite theory assumes that the organisms are so small as to be beyond the range of microscopic vision, but that the virus they produce suffices to provoke that cell-proliferation which is so characteristic of the growth. Such a view does not appear to advance matters very much, for the supposed virus has not been isolated, nor have the organisms credited with its production been procured. It may well be that a stimulus of a chemical nature underlies the whole process; indeed, it is difficult to escape the conviction that it must do so. But the first useful stage in the inquiry would seem to consist in the obtaining of a definite conception as to the nature of the cellular changes themselves.

A second theory assumes, as its foundation, the existence of cells that have become displaced, or have withdrawn from active cooperation in the process of building up the organism during the early stages of embryonic life. These may be cells that should have been destined to give rise to the so-called "germinal epithelium," or to ordinary somatic tissues. In any event, they retain the characters and properties of embryonic cells, and are ready to start into new growth when appropriate conditions awaken them from their dormant state. But in such circumstances they are, of course, freed from the correlating influences that should and would have directed and controlled their multiplication. Thus they come to exhibit in a marked degree that potential independence which is, perhaps, possessed by every unit of nucleated protoplasm.

But this view of the existence of latent germs, scattered over the body fails to account for the remarkable nuclear peculiarities presently to be described, and indeed it seems to savour rather of a petitio principii, since it involves the assumption that the very occurrence of a malignant (or other) growth necessarily postulates the pre-existence of a latent germ at any spot at which the disease has made its appearance.

A third view of the nature of cancer, which may perhaps be appropriately designated as the "gametoid" theory, has quite recently been put forward. It is perhaps easily confused with the "embryonic" theory just outlined, but in reality it is essentially different from it. The gametoid theory, whilst taking account of the parasitic nature of the growth, is mainly founded on the discovery of certain quite peculiar nuclear divisions that normally only occur in connection with the formation of the sexual cells, or of their immediate precursors, in the life-history of the organism.

In the first instance it was during an investigation into the cytology of anomalous growths of ferns, on which the present writer, in conjunction with Mr. Moore and Miss L. Digby, was engaged, that certain peculiarities, previously noted, by the two observers first mentioned, on nuclear divisions in epitheliomata assumed an unexpected significance. A renewed investigation of the cytological processes obtaining in malignant growths seemed desirable, and this work was much facilitated by the additional cooperation of Mr. C. E. Walker.

As the nuclear transformations are somewhat complicated it may be well to explain more fully the relevant facts, such as may be demonstrated in any ordinary animal or plant, and then to indicate the bearing of these on nuclear divisions characteristic of malignant growths.

When an ordinary cell of the animal or plant is about to undergo division, certain definite and constantly 
recurring changes become visible in the nucleus. Delicate strands make their appearance, and these finally segment into a number of rod-like or $\mathrm{V}$-shaped bodies termed chromosomes. The number of chromosomes thus appearing in any nucleus of the ordinary body or somatic cells is quite constant for any species of animal or plant, though different species possess different numbers of these chromosomes. In man, for example, there are thirty-two, whilst in a lily there are

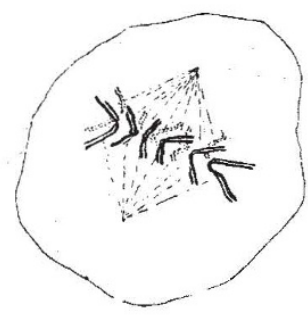

Frg. r,-Normal Somatic Mitosis.

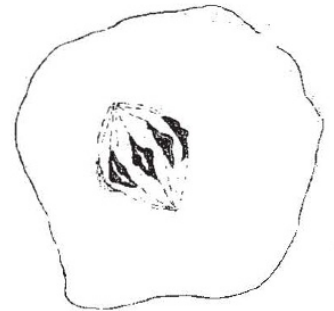

FIG. 2.-Heterotypical Mitosis. twenty-four in all the body- or somatic-cells. The chromosomes become arranged in a very definite manner on a spindle-like structure, and then each of them divides longitudinally into two halves (Fig. I). The two halves then separate, each travelling to opposite poles, and furnish the material out of which the chromosomes of the two daughter nuclei are constituted.

Although the process, as thus given in the barest outline, is constantly met with in all the somatic cells and may be traced back to the earliest divisions in the fertilised egg from which the individual has sprung, a stage sooner or later is reached in the life-history when certain cells become more or less sharply delimited from their fellows, and they finally undergo a nuclear division which is very different in character from that met with in the other cells of the body. To this particular mitosis the term Heterotype has been applied, and its onset marks a radical change that affects the organisation of the descendants of every cell that has passed through it.

The features by which the heterotype can be distinguished from all other mitoses are as follows:The nuclei grow to a relatively larger size, and the strands from which the chromosomes arise exhibit a very characteristic "bunched" appearance at a particular stage in the process. Furthermore, the chromosomes only appear in half the numbers characteristic of the somatic nuclei. In man, for example, where the somatic number of chromosomes is thirty-two, only sixteen appear in the heterotype division. This reduction is due to the cohesion in pairs of the normal chromosomes and not to any elimination of them from the nucleus. The heterotype chromosomes further differ from the somatic ones in form, and this difference is equally marked in both animals and plants. They present the form of rings, loops, \&c. (Fig. 2), instead of the familiar V-shaped figures; and, furthermore, when arrayed on the spindle each divides, not longitudinally but transversely.

Whilst this is not the place to discuss the significance of the remarkable peculiarities that distinguish this heterotype mitosis, it is essential to realise that it marks the point at which the somatic and reproductive elements diverge from each other in their future structure and development. At each succeeding division all the descendants of a cell that has once divided heterotypically retain the reduced number of chromosomes, but in other respects the normal somatic mitoses are NO. I788, voL. 69] closely simulated (Fig. 3). These post-heterotype mitoses are all distinguished as homotype.

Thus the appearance of the heterotype mitosis marks the definite segregation of a sexual series of cell generations. These may be few as in animals, where, after a single homotype division, the sexual elements are at once differentiated. In plants, on the other hand, it commonly happens that all the descendants of the heterotype generation do not actually become differentiated into sexual cells, and in any case the latter are only formed after the occurrence of a number of intervening post-heterotype divisions. These, however, are all characterised by the reduced number of chromosomes (homotype), which, as in animals, is similarly retained in the nuclei of the sexual cells. It is only on the union of ovum and sperm in fertilisation that the full somatic number is restored.

It is perhaps unnecessary to insist that the heterotype mitosis and its consequences are restricted to the reproductive tissue, at least, in the normal body; the somatic cells of the latter, in so far as they continue to divide, present the same features as before.

The general bearing of the foregoing description will become evident when it is stated that both the heterotype and homotype mitoses have been, during the recent investigations, recognised as occurring in certain cells of malignant growths.

If the advancing edge of an actively enlarging tumour, such as an epithelioma, be examined, many cells will be found to be in various stages of division. Near the margin the nuclei commonly exhibit mitoses typical of somatic cells (Fig. I), whilst others will be encountered that show irregularities of various kinds. An excessive number of chromosomes is not uncommon (Fig. 4), and here and there pluripolar figures (Fig. 5) of a remarkable character may be observed. The latter occur somewhat unevenly distributed and owe their
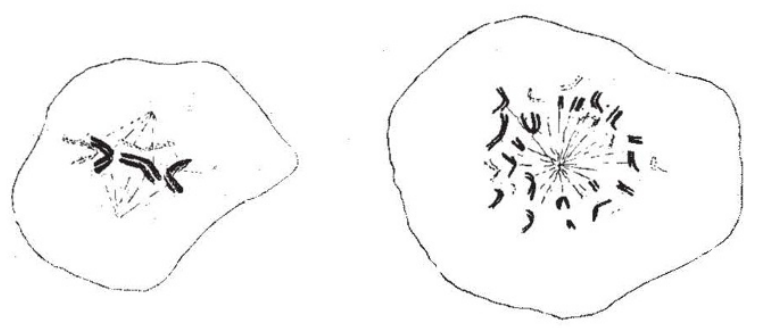

FIc. 3. - Homotypical Mitosis. FIG. 4,-Somatic Mitosis, Polar View,

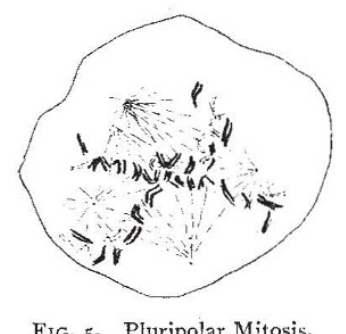

origin, at least in part, to the simultaneous division of a group of adjacent nuclei on a common spindle apparatus. Yet other cells will be met with in which the process of nuclear division is of a type less complex than the normal, and it may be so reduced as to consist in the mere drawing apart of the original nucleus into two similar or even unequal halves, with a more or less complete absence of all differentiation of chromosomes. 
But in spite of these irregularities that tend to obscure the more important facts, the heterotype division can be recognised with certainty in every malignant growth so far examined; and it is precisely similar in character to the normal heterotype that occurs in the sexually reproductive cell series. The same peculiarities in the early differentiation of the chromosomes culminating in the production of rings, loops, \&c., the same reduction in the number, and the same transverse division of each one when attached to the spindle, reappears in these cells with the greatest uniformity.

This peculiar mitosis seems to be confined in tumours to those of a malignant character, for it has not been observed up to the present in any benign growth. It would thus appear to serve as a means of distinguishing between the two classes of growths.

Following upon the heterotype division, the homotype stage is reached, but it very soon becomes unrecognisable in most cases owing to the occurrence of the irregularities above mentioned.

The conclusion to be drawn from the above account is that, in a most important respect, some of the cells of a malignant growth have gone through a change similar to that which in normal tissues is confined to the production of the generations ending with the formation of the sexual cells. Such a conclusion is further supported by considerations derived from other sources.

It has already been pointed out that whereas in animals the differentiation of the sexual elements follows closely after the occurrence of the heterotype mitosis, this is not the case in most plants. Thus in a fern, the whole prothallium is composed of post-heterotype cells, and the sexual elements only arise from a relatively small number of them. Similarly in the embryosac of a flowering plant, there are certain postheterotype cells that are not normally destined to give rise to sexual structures. But it is a matter of considerable interest to find that cells that fail in this respect not seldom exhibit marked irregularities in their modes of further division. Sometimes direct fission of the nuclei may occur with suppression of chromosome differentiation; in other cases the chromosomes may appear, but in quite irregular numbers.

The similarity of these irregularities to those already indicated as present in cancerous growths will at once be obvious from what has already been said.

The investigations of Bashford and Murray have served to confirm the statements previously made as to the occurrence of heterotype and homotype mitoses in the human subject. These investigators have identified the same divisions in malignant growths that occur in other mammals, in reptiles, and in fish. Whether, therefore, the explanation advanced to explain them, which involves the admission of an essential similarity as existing between the malignant growths and sexual reproductive tissue, be accepted or not, it is a fact that will have to be reckoned with.

It has been held by some persons that a transformation of somatic into reproductive tissues cannot occur, and it is, therefore, necessary to examine briefly the grounds on which such an opinion rests.

In plants the difficulty does not really arise, for a large number of cases are known in which cells that have long discharged somatic functions may revert to an embryonic condition, and then, after a heterotype division, produce from amongst their descendants the sexual elements that take part in fertilisation. This fact robs the objection of any a priori force it might have had. It is, however, true that amongst animals the conversion does not normally occur, but the existence of the diagnostic mitosis described above as appearing in the malignant growths affords cogent evidence for NO. I788, vOL. 697 regarding them as representing such a changed condition, the true nature of which is, however, masked by the invariably pathological features that accompany it.

It is not urged that the cancer cells are functionally active sexual elements, but rather that they are homologous with such; it has, therefore, been proposed to express this idea by applying the term "gametoid" to them.

But whilst the existence of the heterotype mitosis emphasises the gametoid nature of the cells that have just passed through it, there are other phenomena that suggest the interpretation may possibly be carried on to another and further stage. Just as the true gametes (sexual cells) may fuse, so, too, cases of nuclear fusion are not very uncommon in the post-heterotype cells of malignant growths. It would be premature at the present juncture to attempt to do more than indicate that there may be something beyond a mere abnormality latent in these fusions. It is, however, a fact that in individual cases the fusion figures strongly recall instances of normal fertilisation. Should the suggestion turn out to be well founded, and many instances apparently support it, much that is still difficult of explanation will immediately become clear. The irregular nuclear divisions, for example, will be no more surprising than are those so frequently to be seen in the endosperm of an angiosperm, or even in the more abnormal results consequent on polyspermy. The independence of the neoplasm and its parasitic habit, to which attention has already been directed, would be still quite explicable, for in a general sense it may be stated that a new generation habitually preys on its forbears whenever continued association with them admits of it.

But the problems that especially invite attack are those concerned with the causes of the transformation of somatic, into reproductive, cells and tissues. These fall within the scope of the physiological chemistry of the cell. Something has already been done in this direction so far as plants are concerned; and, indeed, it would seem that the lower members of the vegetable kingdom offer a more convenient material for investigation than animals. They are comparatively easy subjects of experiment, and their simpler specialisation avoids the difficulties consequent on the presence of complicated subsidiary mechanisms. The ease with which Spirogyra, for example, can be directed into either the reproductive or the vegetative phase is a case in point, and it is only one out of many that could be cited.

J. B. FARMER.

\section{SCIENCE AND MILITARY EDUCATION.}

THE Journal of the Royal United Service Institution for January contains a full account of the important discussion on November 9, 1903, initiated by Lieut.-Colonel F. N. Maude, late R.E., on the subject of military education, and on January i 8 there was published a revised scheme of subjects for the entrance examinations to the Royal Military Academy and the Royal Military College respectively. The discussion at the United Service Institution, which was of a decidedly discursive character, dealt to a large extent with a real or supposed deterioration of the public school boy of to-day, or at least of those public school boys who desire to obtain commissions in His Majesty's Army.

This part of the discussion was based very largely on statements made by army tutors, which, though there may be some truth in them, must be rather carefully scrutinised. First, because army tutors are human, 